\title{
TRAS LOS “DESAPARECIDOS” DE LA GUERRA CIVIL. Una etnografía multisituada y multitemporal
}

Ignacio FERNÁNDEZ DE MATA

Universidad de Burgos igfernan@ubu.es

\begin{abstract}
AFTER THE TRACE OF THE CIVIL WAR'S 'DISAPPEARED'. A multi-temporal and multi-sited ethnography
\end{abstract}

Resumen: El desarrollo del proyecto de investigación Lloros vueltos puños. El conflicto de los 'desaparecidos' y vencidos de la guerra civil española, centrado en el movimiento social por la recuperación de la memoria histórica, ha precisado de una combinación de metodologías que atendiera la irrupción y variedad de contextos y materiales que iban surgiendo. La particularidad de enfrentar un sufrimiento social sucedido en un pasado distante suponía enfrentar comunidades dispersas, espacios marginales de muerte -las fosas-, la configuración de agrupaciones y asociaciones de víctimas..., todo lo cual ha exigido, entre otras, la adopción de metodologías multisituacionales. Inevitablemente, la suma de voces y generaciones conllevó el entremezclamiento de contextos históricos y culturas políticas, lo que requirió adoptar, también, análisis multitemporales. Este trabajo recorre los principales retos metodológicos que caracterizaron el seguimiento del tema de la memoria histórica durante más de una década. El resultado es una reflexión sobre los beneficios o perjuicios de determinadas tareas investigativas en contextos inestables o en rápida transformación.

\footnotetext{
Abstract: The research behind the book Lloros vueltos puños. El conflicto de los 'desaparecidos' $y$ vencidos de la guerra civil española, centered on the social movement for the recovery of historical memory in Spain, was based on a combination of methodologies that could address the irruption and variety of contexts and materials that arose continually and from multiple spaces. Addressing a social suffering that took place in the distant past meant facing dispersed communities, marginal spaces of death-the unmarked mass graves-the configuration of victims' associations and groups..., all of which required the adoption of multi-situational methodologies. The sum of voices and generations meant that historical contexts and political cultures were inevitably threaded and mixed, and the subsequent adoption of multi-temporal analyses. This article elaborates the main methodological challenges faced during the decade-long pursuit of the subject of historical memory. It is the result of a profound reflection on the benefits and costs of certain tasks related to anthropological research in unstable or rapidly changing social contexts.
}

Palabras clave: Memoria histórica. Problemas metodológicos. Etnografía multisituada. Multitemporalidad Historical Memory. Methodological problems. Multi-sited ethnography. Multi-temporality 


\section{Introducción}

El presente texto trata de reunir algunas de las reflexiones metodológicas que han estado presentes durante el trabajo de campo del proyecto de investigación Lloros vueltos puños. El conflicto de los 'desaparecidos' y vencidos de la Guerra Civil española, que con el mismo título ha sido publicado por Comares (Fernández de Mata, 2016).

La investigación ha ocupado unos trece años a partir de lo que fue la irrupción del movimiento memorialista encabezado por la Asociación para la Recuperación de la Memoria Histórica (en adelante ARMH). Para el trabajo de campo, el proyecto se ha enfocado en la provincia de Burgos, con incursiones en otros lugares, fundamentalmente de Castilla y León.

Metodológicamente la investigación ha evolucionado en función de cómo cambiaban también los datos o el campo. La visibilidad que han ido adquiriendo estos colectivos y las tareas de exhumación han producido también cambios en el acceso a la información, que en los primeros años resultaba muy delicada y en ocasiones, conflictiva. Así, al principio, estuvo muy circunscrita al espacio de Aranda de Duero y localidades aledañas de la Ribera con un acceso -no siempre fácil- a informantes de primera generación afectada. Un trabajo que facilitaba acotar los sucesos históricos y su contexto, relacionar a los implicados y sus relatos, con un mayor control de ciertas informaciones y una buena base documental y archivística. Este caso me permitió integrar visiones complementarias y opuestas, conocer las reacciones de los afectados -víctimas/familiares de desaparecidos-, las opiniones de vecinos no afectados -en algunos casos cómplices con su silencio, bystanders ${ }^{1}$; en otros, generaciones recientes, completamente ignorantes de los acontecimientos-, y también las versiones de varios perpetradores -directamente asesinos o muy comprometidos con los hechos del golpe y la guerra-. La incorporación de estas visiones me permitió posteriormente poder trabajar sobre cuestiones relativas a la culpa y el remordimiento de los perpetradores².

Contar con una suerte de grupo, de comunidad accesible -las familias arandinas-, ha permitido también poder ver los profundos cambios en la percepción de sus propias memorias durante el proceso de estudio. Así, la investigación se ha desarrollado entre lo que podríamos denominar una metodología primera más clásica y posteriores enfoques que exigían "seguir las tramas" o "los cuerpos" (Ferrándiz, 2011: 209): una etnografía multisituada y más flexible.

Además de la multilocalidad de víctimas, fosas, asociaciones y otros actores, el proyecto también se ha enfrentado a una cierta multitemporalidad que impregna testimonios y análisis. El pasado nacional y el pasado familiar han supuesto espacios de intelección y presencia, de generación de identidad y convicción política, en ocasiones de cierta confusión. No ha sido infrecuente, por ejemplo, encontrar casos en los que se incurre en claras proyecciones del presente sobre el pasado produciendo efectos distorsionantes. El trabajo sobre las cuestiones de la memoria ha necesitado de un buen manejo de las técnicas de historia oral, del análisis minucioso de los testimonios y de una distancia emocional, difícil en muchos casos, respecto a lo recogido.

1 En psicología se conoce el efecto cómplice, más conocido por su expresión anglosajona "bystander effect". Básicamente, tal expresión viene a decir que, ante la presencia de terceros, se produce una inhibición en aquellos que contemplan una desgracia o una injusticia, quienes no actúan en auxilio de las víctimas. Con ello se contribuye al reforzamiento de la idea de castigo colectivo y, por tanto, complicidad con los que ejercen la violencia. Véase Hudson y Bruckman, A. S. (2004: 168).

2 Partir de un área concreta, en una suerte de estudio de caso, permitió plantear la mayor parte de los problemas de la investigación y contar con informaciones que fueron determinantes para poder ampliar posteriormente el estudio a otras áreas.

Para lo relativo a la fijación de los problemas de la investigación y las muestras dentro de un caso, véase Hammersley \& Atkinson, 1994: 46-67. Igualmente, Ferrándiz, 2011: 42-49. 


\section{Arranque}

Cuando en el año 2003 el ayuntamiento de Aranda de Duero se dirigió a la Universidad de Burgos solicitando ayuda para exhumar las fosas del monte Costaján, ya habían tenido lugar las primeras acciones de la ARMH en el Bierzo (León) y también, un año antes, se había exhumado la fosa de los vecinos de Caleruega (Burgos). Aquellos casos habían sido objeto de una gran atención mediática y producido una gran agitación entre los sectores más conservadores de la sociedad. En ambos casos, su apertura respondía a la acción directa de familiares. En Aranda de Duero, las fosas del monte Costaján se iban a acometer por iniciativa municipal al interferir aquellas en la construcción de una urbanización de chalets allí proyectada.

Esta fue mi entrada en el campo: a demanda. No había aún una asociación local asentada, no existían colectivos de familiares organizados, ni siquiera una lista clara de afectados. Además de las fosas de Costaján, Aranda estaba rodeada de otras fosas -en realidad, toda la ribera del Duero-, lo que dificultaba saber quiénes podían haber sido enterrados en cada una.

En un primer momento me mostré muy preocupado por la "autenticidad" de los datos históricos -cifras, fechas...-, pero enseguida me di cuenta de que mi trabajo debía ser otro, y que lo que debía estudiar era el conflicto personal, el drama íntimo, frente a datos que iban a estar siempre sometidos a la sospecha y la guerra de versiones. No me iba a dedicar a contabilizar las víctimas, sino a comprenderlas. No me enfocaría en hacer un listado de horrores, ni un obituario -para lo que, además, ya había gente dispuesta-. Queriendo conocer los hechos, me interesaba un estudio cualitativo, no cuantitativo.

Obviamente, la atención a la parte diacrónica, relativa a la historia y a su memoria, ha venido desarrollándose a lo largo de todo el proyecto de investigación. El manejo de la bibliografía historiográfica sobre la represión ha permitido ver también su evolución, incremento y, sobre todo, la inclusión de perspectivas antropológicas y otros debates internacionales que irrumpían en la historiografía ${ }^{3}$.

Para ampliar el espectro de solicitantes -a partir de ese momento, informantes- utilicé varios porteros o gatekeepers (Ferrándiz, 2011:73). En aquel momento había en Aranda de Duero dos personas que se habían destacado en la promoción de estas exhumaciones y a través de ambos conseguí incrementar notablemente el número de testimonios/demandas de familias.

Antes de meterme con las entrevistas, comencé a trabajar la literatura relativa a las fuentes orales; por tanto, a realizar un diseño de investigación y fijación de primeros problemas. Más tarde, en el verano/otoño de aquel año, incrementé mis lecturas y reflexiones en la Universidad de Wisconsin-Madison (EE.UU.). Esta estancia de investigación fue fundamental para el desarrollo del proyecto y, sobre todo, para profundizar en los problemas y conflic-

\footnotetext{
3 Es el caso de la irrupción de las controversias relativas a "los usos políticos del pasado". Esta expresión procedía de la acuñada por Habermas sobre el "uso público de la historia". Durante los años 80 se desarrolló en la RFA una disputa entre historiadores -Historikerstreit- sobre el devenir político de Alemania durante el nazismo, su surgimiento y ascenso en la década de los años 30. Este debate reverdeció con fuerza a comienzos del siglo XXI a raíz de un premio literario a Ernest Nolte, revisionista que polemizó con Habermas. Habermas defendía “(...) la comprensión ha de ser más una toma de posición moral y un debate ético-político, incluido en el régimen de la "esfera pública", que una disputa científica sobre el pasado alemán desplegada en el ámbito estricto de la historiografía. Al distinguir claramente los dos planos del discurso (el político y el científico), Habermas argumentaba la oposición existente entre ambas y la desconfianza que debían generar a los especialistas la utilización instrumental y las manipulaciones derivadas del "uso público de la historia". Peiró Martín, 2004: 248 y 249. Un poco después vino la controversia Goldhagen, a raíz de recibir en Alemania el premio Democracia por su libro Los alemanes corrientes y el Holocausto (Goldhagen, 1997), centrado en una extensión de las culpas al conjunto de Alemania frente al manido discurso negacionista del "no sabíamos nada" que ya contestara Jaspers al acabar la guerra mundial.

Estos debates pronto tuvieron su correlato en España, especialmente a partir de la publicación en 2003 del libro Usos públicos de la historia (Carreras \& Forcadell, 2003).
} 
tos de las llamadas fuentes orales que, obviamente desde un punto de vista antropológico social, no podían ser tratadas como meros datos/fuentes de valor historiográfico -como así pretendían algunos amateurs-, era necesario aplicarles una adecuada metodología analítica.

Igualmente, en Wisconsin, profundicé sobre todo en el conocimiento de la Guerra Civil española y el franquismo en el contexto de los distintos totalitarismos del siglo XX, lo que me permitió también acercarme a las historias de las víctimas del nazismo, el fascismo y el estalinismo. Pude, igualmente, colectar y conocer casos relativos a otras guerras civiles europeas, asiáticas y genocidios contemporáneos.

Con tales mimbres resultaba más plausible definir los temas de investigación y las estrategias primeras. Era el momento de planificar "la hoja de ruta". Fue así como me concentré en la preparación de entrevistas intensivas que, por el tema y participantes, participaban en cierta forma en un concepto de historia de vida ${ }^{4}$.

\section{La entrevista}

Teniendo en cuenta la estructuración del proyecto, la mejor vía para abordar esta fase era la de definir un detallado cuestionario que me permitiera obtener una información estructurada, que facilitara la organización de los datos y, si fuera necesario, su contraste. Necesitaba contar con la mayor cantidad de referencias posibles para cumplir con los objetivos de las identificaciones, del conocimiento de los hechos y de la experiencia de las familias. El cuestionario fue pensado también como proceso de acercamiento y negociación, en el sentido de dar entrada a los temas más conflictivos después de haber recorrido contextos más globales, en los que se podían evidenciar ideologías, sociabilidad, percepción de la política del presente... Como estrategia trataba de conseguir lo mismo que Ruiz Olabuénaga sugiere empleando una pregunta general o lanzadera, procediendo "de fuera a dentro y de dentro a fuera", es decir, de lo general a lo singular y de lo de menor a mayor complejidad; pero sobre todo buscaba romper el hielo y crear "una buena relación interpersonal personal en la que los participantes responden y facilitan información sobre sus experiencias personales" (Ruiz Olabuénaga, 2003: 187 y 166).

El lugar. Para un mejor desarrollo de las entrevistas siempre procuré que éstas fueran en los domicilios de los demandantes. Esto, por un lado, facilitaba las cosas al tratarse mayoritariamente de personas muy mayores, de manera que podíamos soslayar cualquier dificultad física o de salud. Por otro lado, que las conversaciones se mantuvieran en el marco de seguridad de sus hogares, les permitía encararlas con un pequeño grado de confianza mayor pues les resultaba muy difícil hablar de experiencias tan íntimas y dolorosas con un extraño, máxime después de haber tenido que ocultarlas -silenciarlas- durante tantos años.

Siempre me mantuve muy atento a la elección del espacio que hacían mis informantes dentro de su propia casa: cómo elegían representarse, en tal o cual habitación, rodeados de ciertas fotografías o elementos que para ellos tenían especial significación o valor. Sabía que a través de aquellas imágenes y rincones trataban de construir su historia e imagen. En la mayoría de ocasiones, había una persona de apoyo con el/la informante, generalmente un

\footnotetext{
4 Charriez Cordero, 2012: 50-67. Pujadas, J. J. 1992. El método biográfico. El uso de las historias de vida en ciencias sociales. Madrid: CIS. En este caso, siguiendo más el modelo de life story. No fue, sin embargo, un modelo fidedigno del desarrollado por Pujadas que sugiere una actuación más abierta, apoyada en documentos personales (cartas, fotografías, diarios personales), aun cuando éstos estuvieron presentes en las entrevistas, aportados por los entrevistados y abriendo, así nuevos horizontes informativos. Esta forma de trabajar la recoge con detalle en Pujadas, J. J. 2000. "El método biográfico y los géneros de la memoria”. Revista de Antropología Social, 9: 127-158. Aquí, pág. 139.

También: Del Río Sánchez, A. - Valcuende del Río, J.M. 2008. "Historias de vida y microbiografías. Una aproximación metodológica”. En Acosta Bono, G. - Del Río Sánchez, A. - Valcuende del Río, J. M. (Coords). 2008. La recuperación de la memoria histórica. Una perspectiva transversal desde las ciencias sociales. Sevilla: Fundación Pública Andaluza Centro de Estudios Andaluces.
} 
hijo, cónyuge y en algunos casos, alguna amiga, que se sentaba cerca o guardando linealidad con la mirada del informante. Evidentemente eran personas conocedoras de las historias y del sufrimiento y, generalmente, prestaban sobre todo apoyo emocional, v.gr. cogiéndoles las manos o abrazándoles en los momentos de máxima intensidad de su relato. Rara vez estas personas de apoyo intervinieron -salvo en el caso de cónyuges, con amplio conocimiento o vivencia de los hechos-. Ha de tenerse en cuenta que estamos hablando del comienzo del proceso exhumatorio, con todos los miedos en alto, y que estas familias se encontraron casi repentinamente con la oportunidad de exhumar sin que hubiera mediado clara demanda pública por su parte o afiliación a asociación alguna. Su suerte cambió repentinamente al albur de una actuación municipal.

Evité en todo momento acordar entrevistas con la presencia de alguno de los porteros. Las pocas veces que me vi obligado a hacer alguna entrevista repentina, casi impuesta, al presentarme a algún informante, los resultados fueron malos y procuré repetirla posteriormente. Sucedía que por parte del introductor había un claro marcaje al informante, con intromisión en su relato -acabándole las frases, llenando los silencios, indicándole qué contar, valorando o enjuiciando el testimonio ${ }^{5} \ldots-$.

El cuestionario base de la entrevista se concibió como una guía que facilitara la obtención y organización de los datos, un apoyo que permitiera conversar con los informantes con un diseño de información por bloques. Como no sabíamos el acceso que íbamos a tener a los afectados, fue concebido de forma que también pudiera ser fácilmente contestado por escrito en caso de necesitar ser enviado. En cualquier caso, se planteó siguiendo el modelo de "entrevista reflexiva" (Hammersley \& Atkinson, 1994: 128) y con un sentido cronológico -life story-.

En cuanto a su estructura, comienza con los datos del "solicitante", un apartado de identificación de los reclamantes que permitiera ir esbozando el esquema familiar de las víctimas. A continuación, se centra en los detalles biográficos del desaparecido, en lo posible antes de Guerra: rastreamos su sociabilidad, amistades y relaciones, espacios de ocio, contexto sociopolítico, situación de la familia, conflictividad... Todo lo que facilite el retrato de la víctima: preferencias y querencias, trabajos y empeños, militancias, ideologías, participación en protestas o propuestas sindicales o políticas, formación y lecturas, creencias, entreverándolo con la vida social del barrio, parroquias, ayuntamiento, casa del pueblo, sindicatos... Después nos metíamos con los hechos más dramáticos del prendimiento y asesinato.

Dado que lo más usual era que mis informantes y yo no nos conociéramos previamente a mi visita, o que hubiéramos sido brevemente presentados y quedado para una ulterior visita, mi llegada a las casas solía generar una evidente tensión. Estas personas, en su mayoría muy mayores, sabían perfectamente de qué íbamos a hablar, y del sufrimiento que subyacía en aquellas informaciones. Desconfiaban de que tras décadas de oprobio o desprecio por aquellos datos ahora un extraño se llegara a sus domicilios interesado en sus vidas. En su inmensa mayoría eran personas muy humildes, con formación básica que, a pesar de haber deseado denodadamente la resolución de su conflicto, se sentían en medio de un extraño juego entre el ayuntamiento y la universidad -esta última una instancia por completo ajena a sus vidas-.

Iniciar las entrevistas con aquella veintena de preguntas previas sobre contextos y ubicaciones buscaba generar un clima y relación de escucha que sirviera para reducir la tensión de los informantes. Esta era una fase que podía extenderse más o menos en función de cómo

5 "Garanticen o no la entrada al lugar, a los porteros, generalmente, les interesará, comprensiblemente, dar una imagen de la organización que el etnógrafo va a retratar, y tendrán intereses prácticos en que a ellos y a sus colegas se les presente bajo una luz favorable. Como mínimo, ellos desearán salvaguardar lo que perciben como sus intereses legítimos. Los porteros, por lo tanto, suelen intentar ejercitar algún grado de vigilancia y control, tato para bloquear ciertas líneas de investigación como para guiar al trabajador de campo en una dirección u otra". Hammersley \& Atkinson, 1994: 80. 
se produjeran los prolegómenos a la propia entrevista, en los que las normas de hospitalidad y cortesía obligaban a detenerse en comentarios sobre la casa, la salud y situaciones de las personas, a ofrecer y tomar un café o un vino... y responder a las preguntas más o menos directas sobre la persona del entrevistador, su familia, su orientación política, para qué iban a servir las preguntas, qué se pensaba hacer, si sus nombres iban a aparecer en periódicos o revistas... Un justo quid pro quo que generalmente facilitaba el intercambio de información ulterior.

Ya sentados en el espacio elegido por el/la anfitrión, acordado que usaría una grabadora -siempre situada fuera de la línea visual directa del informante-, acompañándome de una libreta en la que iba anotando, iniciábamos la entrevista de la manera ya explicada. Con las primeras respuestas solía producirse un acartonamiento del lenguaje, una formalidad oral autoimpuesta ante la situación formal de la entrevista -sentados frente a frente, con aquella grabadora y mis notas de por medio-. Siempre adapté las preguntas a las fórmulas conversacionales en las que habíamos comenzado mi visita, dejando que si mi interlocutor elegía un camino diferente o deseaba ampliar alguna información lo hiciera. Conseguía volver a la coloquialidad y tonos previos. La apoyatura del cuestionario me permitía mantener un guion mental de la información buscada y facilitaba reconducir la conversación hacia la sección que faltara o hubiéramos saltado.

Tras la primera veintena de cuestiones contextuales, y habiendo permitido las posibles derivas previas -"relatos no solicitados" (Hammersley \& Atkinson, 1994: 123)-, nos centrábamos en lo sucedido al/los desaparecido/s. Eran los momentos más tensos de todas las entrevistas. Empezábamos tratando de reconstruir los recuerdos del 17 de julio de 1936 para ir entrando en el nuevo tiempo de la guerra, los cambios sociopolíticos experimentados en la villa. Entonces acometíamos el prendimiento de las víctimas, momentos desgarradores que solían suceder en el hogar, a horas intempestivas de la noche. Irrumpían relatos de niños que veían desaparecer a sus padres, que conservaban congelados los instantes de los golpes en las puertas, la irrupción de los falangistas o los guardias civiles, la despedida... A partir de este punto el relato solía manifestarse narrativamente más seguro, reafirmado por la experiencia y la intensidad del recuerdo traumático. El cuidado en la prisión, las cartas recibidas, visitas para llevar alimentos, mantas, ropas, hasta que un día se les informaba que ya no estaba allí. Toda esta sección se desarrollaba de forma angustiosa y era habitual que los informantes lloraran desconsoladamente. Eran momentos cruciales, de irrupción de sus penas y sentidos de pérdida que dejaban de ser algo teórico y sobreentendido para mostrarse con toda su crudeza y dolor. Situaciones intensas que pusieron en jaque todos mis juicios y preconcepciones sobre la implicación personal. Aquellos instantes críticos se resolvían con la ejecución de gestos que les transmitiera mi solidaridad y empatía -agarrándoles, acercándome-, nunca quitándoles yerro o callando. Solía ayudar que nos quedáramos un rato girando en torno a la última idea - una suerte de técnica de "espejo viviente" (Ruíz Olabuénaga, 2003: 182) -, el comentario que había provocado el llanto. Al poco, volvíamos al cuestionario, generalmente por iniciativa de los informantes.

A partir de aquí los bloques de preguntas se centraban en la situación en que quedó la familia, sus sufrimientos, expolios, vejaciones y conflictos durante la guerra, el franquismo y la democracia, sus anhelos y esperanzas. Luego íbamos a la gestión de las exhumaciones, las relaciones con el ayuntamiento, la irrupción de la ARMH, la percepción de la política... También había una parte dedicada a rastrear el odio y la venganza, las relaciones con los perpetradores locales y la percepción de la justicia.

La relación creada con los primeros informantes, gentes en muchos casos desprovistas de lo que ellos consideran apoyos sociales eficaces, con una larga experiencia de sufrimiento y subordinación, convirtieron nuestra relación en una suerte de reafirmación que rápidamente entendieron como un cambio de percepción social sobre ellos. Comprendieron que con su colaboración en mi trabajo iba implícita la reivindicación de su situación y la dignificación 
de sus allegados asesinados -veníamos de la universidad a conocer sus casos...-. Una vez realizada la primera decena de entrevistas, fue habitual que me recibieran diciéndome "ya estaba yo esperando su llamada..." o "ya me extrañaba a mí que no me llamara...". Se había generado una demanda de atención y deseaban ser escuchados y comprendidos. Entramos en un tipo de relación que iba más allá de la entrevista. A partir de entonces yo me convertía en su "fuente" de provisión de documentos, especialmente de los archivos, de todo lo cual me pedían copia. Ellos me habían provisto de fotos, cartas, algún documento de los registros civiles... Se encadenaba así toda una serie de relaciones de reciprocidad que, “(...) son ámbitos complejos y ambivalentes, saturados de tensiones, de manipulación, de diferencias extremas de poder, y de injusticia. Pero también son ámbitos que permiten y sustentan la ayuda mutua y las transferencias de recursos" (Narotzky, 2002: 28). Todo ello, además de colaborar con mis informantes ayudaba a que pudiera revisitarlos, contrastar datos, hacer nuevas preguntas.

Esta es solo una parte de las tareas de campo. Otra parte importante de la investigación se desarrolló, como no podía ser de otra manera, a pie de fosa.

\section{En las fosas}

Hasta aquel momento no había tenido una relación intensa con las fosas. En Costaján apenas tuve presencia en la fosa, celosamente custodiada por los arqueólogos bajo discutibles criterios de seguridad laboral. Aquello dificultó enormemente el acceso a la exhumación, tanto a nosotros como a los familiares, por lo que socialmente no fue un espacio de trabajo y relaciones.

Mientras el proyecto de investigación iba tomando forma, el compromiso también lo hizo. 2003 fue un año de actividades frenéticas, entre las que estuvo un curso de verano que tuvo cierta repercusión y que conllevó la creación de la Coordinadora para la Recuperación de la Memoria Histórica de Burgos.

La Coordinadora nacía independiente de las agrupaciones que ya venían funcionando y definió la provincia de Burgos como su ámbito de actuación. Comenzó sus reuniones en un local prestado del edificio que comparten en Burgos los sindicatos UGT y CC.OO. A partir de aquel momento, las reuniones de los primeros jueves de cada mes se convertirían en el espacio de recepción de familiares que deseaban solicitar que se exhumaran sus fosas comunes. Todo empezó como un goteo. El grupo fundador pivotó en torno a investigadores y un puñado de familiares. Pronto los investigadores dimos un paso atrás para que fueran los familiares quienes se hicieran cargo del día a día. Esta fue una decisión meditada y buscada: debían ser las familias quienes gestionaran sus reclamaciones -y era preciso generar ciertas distancias para el mejor desarrollo de la investigación-.

Se hizo necesaria un nuevo diseño de investigación: una metodología multisituada que me permitiera un tipo de observación y análisis adaptados a condiciones, espacios y sujetos cambiantes. Dada la existencia de otras asociaciones en la provincia, muy centradas en una fosa o área comarcal concreta, había de asistir a exhumaciones de las que no conocía todo el trasfondo de su gestión lo que llevaba a plantearse también de manera mucho más explícita que el aquí y ahora de la exhumación. Contaba con una cierta comunidad en torno a la Coordinadora, pero también sujetos en movimiento, que entraban y salían en el grupo para la resolución de sus conflictos, convergentes temporales.

El trabajo de campo, el campo en sí, como señala Laplantine, no tiene "existencia propia, sino que siempre resulta de la confrontación entre el etnógrafo y el grupo social y cultural, haciendo del campo el resultado de esta interacción"6.

6 Citado por Dumont, 2012: 70.

Por otro lado, "el trabajo de campo es sobre todo una forma de escritura, está impregnado de subjetividad -la ocultemos o la exhibamos-, se funda sobre cronotopos específicos en los que se cruzan el tiempo del relato y el 
Marcus señala cómo en la etnografía multisituada el estudio "se efectúa a partir de plantear preguntas a un objeto de estudio emergente, cuyos contornos, sitios y relaciones no son conocidos de antemano (...). El objeto de estudio es en última instancia móvil y múltiplemente situado" (Marcus, 2001: 115). La característica de esta forma de trabajo será, inevitablemente, la flexibilidad: Entendía que aquellas iban a ser las coordenadas de mi trabajo y que, la investigación había de diseñarse “(...) alrededor de cadenas, sendas, tramas, conjunciones o yuxtaposiciones de locaciones en las cuales el etnógrafo establece alguna forma de presencia, literal o física, con una lógica explícita de asociación o conexión entre sitios que de hecho definen el argumento de la etnografía" (Marcus, 2001: 118). En definitiva, según Marcus, la etnografía multisituada define como objeto de estudio los fenómenos sociales que no están centrados en un solo lugar y que por tanto no pueden encararse sino es desplazándose en un espacio cambiante e incierto ${ }^{7}$. O como proponen Nadai y Maeder, atendiendo no tanto a los distintos mundos sociales en los cuales los individuos permanecen, sino examinando un mismo mundo social a través de sus multiplicidades espaciales ${ }^{8}$.

La forma en que he enfocado mi trabajado de campo sobre las fosas ha sido fundamentalmente observacional. En este tipo de investigación hay una parte del trabajo de campo que se repite en cada exhumación, que es un permanente nuevo comienzo con cada nueva fosa. Inevitablemente, cuestiones clásicas del trabajo de campo, como la rutinización de nuestra presencia, han de ser resueltas de otra forma y con otros enfoques. Una exhumación es un locus investigativo complicado.

Si tomamos como referente la tipología de distintas observaciones y participaciones propuestas por Dewalt \& Dewalt (2002) -siguiendo a Spradley- mi trabajo se sitúa entre las posibilidades dos y tres, entre la participación pasiva y la moderada ${ }^{9}$. Opté por tener un perfil bajo que me permitiera deambular entre la gente, observar y escuchar con atención las reacciones, comentarios, la conformación de grupos... Todo muy abierto, respondiendo, en definitiva a lo que Ferrándiz señala como "un proceso metodológico relativamente desestructurado mediante el cual un observador toma parte en las actividades cotidianas, en los rituales, en las interacciones, en los sucesos en los que participa la gente estudiada, con el fin de aprender los aspectos explícitos e implícitos de la cultura" (Ferrándiz, 2011: 87).

En mi caso siempre he tratado de hacer sentir cómodos a mis informantes con mis actitudes, entradas, vestimenta... buscando el "lenguaje silencioso" de Hall y Hall, facilitando la empatía para reducir los inconvenientes o distancias, pero siempre sin disfrazar mi identidad u ocultar mi condición universitaria y ciudadana. Creo que esta actitud me ha evitado muchos conflictos y, sobre todo, me ha ayudado a solucionar prevenciones o prejuicios sobre mi persona. Desde luego, en el arranque de la misma, con las entrevistas intensivas, resultó particularmente positiva.

He practicado pocas entrevistas intensivas a pie de fosa. La complejidad de una exhumación, con las emociones a flor de piel, cierta exaltación, habitual presencia de gentes con ganas de dar discursos políticos, mucha atención mediática, etc., convierten a éste en un

tiempo nativo, o sobre estrategias narrativas a veces perversas. Es una representación del Otro que lo deforma o lo congela, lo somete o lo cosifica, pero que siempre es construida siguiendo una serie de convenciones distintivas del género etnográfico, que se corresponden con lo que Marcus y Cushman -entre otros- han llamado el "realismo etnográfico" (Marcus \& Fischer, 2000: 50-51). El antropólogo no sólo escribe. El trabajo de campo es narrativa y es también experiencia”. (Cantón Delgado, 2008: 149).

7 Falzón, 2009: 1.

8 Citado en Dumont, 2012: 71.

9 Las cinco posibilidades son: 1) La "no participación”. Utiliza los medios de comunicación, la literatura, el arte... 2) La "participación pasiva": es la que se produce cuando el investigador está en el terreno y observa sin interaccionar con la gente. Los observados pueden no ser conscientes de la presencia del antropólogo. 3) La "participación moderada". El investigador participa de forma limitada, con conocimiento del personal. 4) "Participación activa”. El grado de implicación es superior y se produce una mayor integración en el grupo. 5) "Participación completa", con total implicación e identificación con sus sujetos de estudio. Dewalt \& Dewalt, 2002. 
ámbito de muy difícil control. Desde luego he realizado entrevistas y he coordinado equipos de estudiantes con ese objetivo pero la información obtenida es muy variable. Son más útiles para facilitar las identificaciones a los forenses, que para revelar el intenso drama de los deudos de los desaparecidos. No resulta fácil producir un espacio de intimidad suficiente para entrevistar a personas desconocidas con las que es muy difícil utilizar técnicas de entrada a una conversación fluida. Los familiares están fuera de su contexto, culminando una larga espera, sumamente nerviosos. Muchos de los curiosos que se acercan a una exhumación, especialmente los más ideologizados, observan a los familiares a través de un "capital simbólico" atribuido que los transforma en valiosas evidencias de tipo político. Esto despierta un interés por sus movimientos, sus declaraciones, entrevistas... que condiciona enormemente. En cualquier caso, la información que se produce en estos contextos debe ser tratada de forma específica, teniendo muy en cuenta la presión y demanda que se produce sobre los informantes. En no pocas ocasiones, en exhumaciones en las que conocía previamente a los familiares y teníamos relaciones fluidas, se me han acercado alguno de estos tras ser entrevistado por periodistas o incluso por mis estudiantes para decirme que se habían quedado muy a disgusto con lo que habían dicho, que se les había olvidado esto o lo otro, que estaban muy nerviosos... Demasiadas veces las preguntas resultaban incómodas para responder al "aire libre". En todo caso, a pie de fosa, una actitud menos inquisitoria, sin ejercer presión, conduce a una mejor y mayor empatía y liberación.

En este sentido, mis notas de las exhumaciones se centran en una observación muy atenta pero poco invasiva, apoyada en la experiencia de muchas entrevistas y conversaciones previas y en el conocimiento de la gestión previa de la exhumación.

\section{De la observación a la participación: la lobera}

Quedan por aclarar mis roles en las exhumaciones. En este largo periplo de investigación he podido conocer las exhumaciones desde distintos puntos de vista, metodologías y responsabilidades, lo que me ha permitido tener una experiencia más completa del fenómeno. En 2004, por encargo del Ayuntamiento de Aranda de Duero, tuve que ponerme al frente de la exhumación de la fosa de La Lobera, segunda de las exhumadas en la villa ribereña. Esta fosa tenía una gran importancia simbólica para los arandinos por ser $\square$ aparentemente $\square$ la primera que se abrió en 1936. La creencia popular mantenía que allí estaban enterrados miembros de la corporación municipal de entonces, aunque, en realidad, la mayor parte de los concejales asesinados se encontraban en las fosas de La Andaya y Villamayor de los Montes, fruto de sacas de la prisión central de Burgos. La relativa paradoja de que un antropólogo social se hiciera cargo de la coordinación de la exhumación procedía del grave desencuentro que ya se había producido con el responsable de las exhumaciones de Costaján -paleontólogo-, incapaz de incluir el drama de los asesinatos a sus "restos esqueléticos", más enfocado al desarrollo de lo que imaginaba una suerte de start up de exhumaciones. De ahí que el Ayuntamiento se dirigiera a mí para esta segunda campaña arandina ${ }^{10}$.

La exhumación se planteó a partir de los protocolos científicos marcados por el Dr. Francisco Etxeberría, para producir información de carácter rigurosamente pericial. El objetivo era doble: extraer el máximo de información del sitio mediante metodología arqueológica y forense, lo que iba a permitir reconstruir la escena del crimen, y producir información individualizada sobre cada resto esquelético, tanto para conocer las causas de su muerte como para aportar otras evidencias sobre la vida de aquellos asesinados (Etxeberría, 2004).

Participar de las tareas exhumatorias supuso un vínculo especial con las familias. La posibilidad de colaborar en la recuperación de los huesos, de tocarlos reverencialmente como

10 En cualquier caso, una situación que no ha sido excepcional como demuestra, por ejemplo, el caso de Julián Lopez y Francisco Ferrándiz, quienes coordinaron la exhumación de Fontanosas, en Ciudad Real, en 2006. López García \& Ferrándiz, 2010. 
hacen los voluntarios con sus paletas y brochas, supone la identificación total con los sueños de los familiares, captar la intensidad mágica de los huesos y participar de la sensación de alivio que reciben con la apertura de la fosa. Uno llega a una percepción diferente de sí mismo: ya no es únicamente el que hace preguntas; aparece allí, en medio de los huesos, arrodillado con el resto, y las familias extienden aún más su cariño, su complicidad.

En nuestra experiencia directa en Aranda, el cambio de estilo en la gestión de la exhumación -con relación a Costaján- se vio reflejado en una relación mucho más positiva del equipo con la población y las familias en particular. Al igual que venía haciendo la ARMH en otras exhumaciones, en todo momento procuramos que fuera una exhumación visitable. Pusimos carteles informativos, se adaptó el terreno y me encargué personalmente de recibir a todos los visitantes, explicarles qué se hacía y, cuando tenían/querían dar alguna información, atenderles.

La experiencia de gestión de la fosa me reafirmó en la importancia de la investigación antropológico-social para este tema, y en la necesidad de trabajar colaborativamente con arqueólogos y forenses. No era solamente una cuestión de informaciones complementarias (Douglas, 2014). Hacía falta entender los lenguajes, métodos y demandas del resto. La presencia de la antropología social, con la cercanía a las historias y vivencias de las familias resultaba también fundamental para los arqueólogos, acostumbrados a una relación con la tierra y los huesos muy distinta, en ocasiones muy poco sensible. La forma de referirse a los huesos, por ejemplo, cambiaba inmediatamente. "Restos esqueléticos" era una fórmula inadecuada, distante, despersonalizadora. De ahí la cierta paradoja de que habitualmente se hable de "cuerpos". Los restos encontrados tienen identidad -en busca-, nombres y apellidos, historias terribles y familiares presentes. El delicado objeto de angustia para los deudos era construido en su sentido de pérdida total a través del cuidado, interacción y minuciosidad metodológicas. Esta línea de trabajo, en lo que podríamos denominar arqueología social, ha dado frutos interesantes, de entre los primeros, Juan Montero (Montero, 2010 y 2011). Layla Renshaw (Renshaw 2010, 2011 y 2013), sigue esta estela interdisciplinar con una gran atención a los hallazgos arqueológicos, apoyándose en el trabajo Parker Pearson (1999) y Ewa Domanska (2006), interpreta los restos corporales como rastros del pasado usados por los vivos para construir sus representaciones del ayer, el presente y futuro.

En el caso de la exhumación de La Lobera, sin participación de activistas políticos, sabíamos de los conflictos de los familiares y de la construcción de extrañas lógicas para entender la muerte de sus deudos (Fernández de Mata, 2006). Así, entendimos la dificultad que enfrentaban algunas familias para visitar la fosa en aquellos primeros momentos (2004), una mezcla de temor y angustia, de inseguridad, pues aún no se había producido el cambio sobre su percepción social que sobrevino después. Fue por ello que organizamos diversos actos de dignificación de las víctimas, homenajes, y visitas de personalidades políticas. Organizamos también dos ciclos de conferencias sobre lo sucedido y la metodología de análisis que empleamos para nuestro trabajo. Los resultados finales de este trabajo exhumatorio fueron entregados en el mes de junio de 2005 al ayuntamiento de Aranda de Duero. Esta nueva fase de exhumación facilitó encontrar nuevos testimonios de gente tanto residente en la Ribera como emigrados a otros lugares que, de no acercarse ellos a la exhumación, habrían sido muy difíciles de localizar.

Las fosas tienen una tipología diversa. No es lo mismo una fosa pequeña que una grande, una rural que una urbana -y éstas, según quienes sean sus promotores-. Las fosas de los pueblos han tenido una gestión muy comunitaria, con fuertes implicaciones de sujetos que han acabado, en algunos casos, arrastrando al pueblo entero con homenajes multitudinarios y auténticos cierres de conflictos. Las fosas grandes son más complejas y reúnen en torno a sí muchas proyecciones ideológicas. En mi caso, las exhumaciones de las fosas de Estépar han mostrado perfectamente estos juegos y tensiones que llegan a provocar la queja de los familiares. Estépar es un pueblo cercano a la ciudad de Burgos que acoge centenares de 
asesinados, fruto de sacas procedentes del Penal Central de Burgos. Aquí hemos enfrentado uno de los fantasmas de una ciudad tan señalada por la Guerra Civil como Burgos. Estépar es uno de los nombres del horror colectivo interno de Burgos y una seña de identidad de la izquierda que lleva realizando homenajes y visitas anuales a este paraje desde mediados de los años 80. En Estépar están enterrados los políticos e intelectuales burgaleses más comprometidos. Un espacio de hondo -y deseado- simbolismo. Inevitablemente, éstas han sido exhumaciones de una mayor proyección social, con una atención permanente de los medios de comunicación. Este ha sido un espacio muy interesante para la observación y comparación con las primeras exhumaciones, así como para ver el gran cambio de percepción social habido en torno a estas reclamaciones. Además, Estépar ha sido una de las primeras fosas de España en ser financiadas a través de crowfunding. Los organizadores fue el colectivo artístico Espacio Tangente, pintores y fotógrafos en su mayoría, y que desde hace unos años acogen en sus instalaciones las reuniones de la $\mathrm{CRMH}^{11}$.

Otro ámbito de trabajo han sido los homenajes y reentierros, también de muy distinta tipología: muy íntimos en el caso de pueblos y fosas pequeñas, muy públicos en localidades mayores donde se pone en juego un capital simbólico que políticos y grupos quieren utilizar.

\section{Conclusiones}

Como ha podido verse, la multisituacionalidad del trabajo ha atendido, en primer lugar, a las circunstancias que marcan las fosas, radicadas en espacios marginales de condición singular. Situados fuera de los pueblos de origen, fueron concebidos como lugares anonimizados y confusos, ocultos; muchas veces en los márgenes de los términos municipales cuando no invadiendo otros términos vecinos tratando con ello de descontextualizarlos aún más. Son y no son espacios de la comunidad. Dado su origen, la voluntad ocultadora de los perpetradores, y sin que medie juego de palabras, no son espacios de vida, sino de muerte; al menos hasta el momento de la exhumación. Solo entonces la vida bulle momentáneamente.

La apertura de las fosas reúne varias comunidades interactuantes y muy disímiles, objeto de la metodología multisituacional: la de los familiares -habitualmente disgregados hasta ese momento- junto con algunos vecinos, la del equipo de exhumación -responsables y voluntarios-, y la más volátil, la de los curiosos e interesados que se acercan en distintos momentos. Entre todas ellas, se encuentra la comunidad de los asesinados que se visibiliza en sus últimos instantes de vida a través de lo que la arqueología trae a la luz y en la evocación hecha por los familiares de cada uno de ellos, y que emprenderá nuevos desplazamientos -laboratorios arqueológicos y forenses, entregas a familiares, reenterramientos...-. Durante el período de exhumación, las fosas son espacios de conocimiento, de fértil interacción, en la que se producen algunas entrevistas y, sobre todo, mucha observación e intercambio de ciertos datos y contactos.

Obviamente la condición de las víctimas, de las familias de los asesinados, no es estática, máxime cuando han pasado casi ochenta años de los sucesos traumáticos.

La conflictiva localización de los informantes tiene relación con las condiciones de vida sufridas por las víctimas. Mucha gente, a tenor de las terribles experiencias pasadas, abominó de sus pueblos y grupos, rompiendo toda relación ${ }^{12}$. A esto hemos de sumar el resto de migraciones, movimientos y cambio de condiciones vitales de las siguientes generaciones -los grandes desplazamientos de los años 50- que ha hecho que en muchos casos contemos con comunidades virtuales de personas a las que les une su condición de afectados/familiares pero que no participan de una vida común. Las exhumaciones visibilizan una reconfigu-

11 Se obtuvieron $15.350 €$. Recuperado de https://goteo.org/project/monte-de-estepar

Estas exhumaciones no habrían sido posibles sin la participación desinteresada de la Sociedad de Ciencias Aranzadi, posiblemente el mejor equipo arqueológico-forense existente en España.

12 Véase el caso estudiado por Díaz Sánchez, 2016. 
ración de las relaciones del pasado y de algunas de las múltiples identidades que organizan nuestras vidas. Los espacios de las fosas adquieren un importante simbolismo en el que se produce la confluencia de los tiempos -multitemporalidad superpuesta-: el de los asesinados y sus asesinos; el de las generaciones intermedias que han sostenido con su memoria el recuerdo de lo sucedido y el valor del lugar; el de los que consiguen la realización de la exhumación y el cumplimiento de las promesas y cierre de los duelos. De ahí la importancia de la observación durante las exhumaciones.

La incorporación de las siguientes generaciones tuvo repercusiones también en la gestión de las exhumaciones en las que se empezó a ver una mayor participación de familiares, siquiera en tareas auxiliares. Esto ha hecho que, en cierta medida, sí podamos hablar de una cierta comunidad, basada justamente en la compartición de experiencias similares y en la participación solidaria en las exhumaciones. Estas generaciones, duchas en el uso de la tecnología -internet, smartphones, ordenadores...- acababan conformando foros, páginas de Facebook, intercambiaban direcciones de correo electrónico, de twitter... con lo que estrechaban lazos en la configuración de esa suerte de comunidad virtual. Esta es otra parte, no localizable, a la que se ha atendido en la investigación.

Algunos de estos espacios virtuales han sido -son- constitutivos de comunidades muy activas. Las posibilidades que ofrece internet hacen que una parte de nuestra atención se dirija a las redes sociales. Así, una de las tareas permanentes del proyecto en estos últimos años ha tenido que ver con la atención a estas comunidades virtuales. Se trata de un nuevo tipo de observación: la webservation (Varisco, 2000), que inevitablemente interpenetra la realidad, participa de los actos, activa la militancia, localiza a gente y aporta significados.

Por último, en cuanto a metodología investigativa, queda por mencionar la aplicada al estudio de un fenómeno espontáneo que tuvo lugar durante los años 2006 y 2007: la guerra de las esquelas. Volviendo a la clasificación metodológica de Dewalt \& Dewalt, fue ésta una observación pasiva. El inesperado fenómeno tuvo lugar en periódicos tanto de tirada nacional -primero-, como locales -después-. Los textos de afirmación y conclusión ritual de los desaparecidos por represión nacionalista fueron poco después contestados por otras esquelas que devolvieron a la actualidad a otra categoría de víctimas: los Caídos por Dios y por España. Y, a pesar de lo doloroso del fenómeno, desde un punto de vista antropológico fue de gran interés este intento de contraposición de unas víctimas contra otras. La metodología empleada para estas esquelas fue de tipo hermenéutico -por otro lado, de textos multisituados al hallarlos por toda la geografía nacional-. Obviamente, el trabajo sobre estos materiales no podría haberse hecho con la eficacia y profundidad empleadas de no haber contado con la experiencia previa de un trabajo de campo junto a las familias de víctimas, lo que me permitió ajustar mucho mejor cuestiones relativas a la intensidad del drama, la narratividad del sufrimiento y los conflictos de identidad de los colectivos de víctimas implicados.

En definitiva, sin el desarrollo de una metodología multisituacional y una perspectiva multitemporal, no es posible enfrentar este tipo de conflictos que atienden al pasado y al presente, al locus histórico y a la líquida contemporaneidad, a un mundo glocal y abierto que no puede renunciar a sus anclajes y lealtades.

\section{Bibliografía}

Cantón Delgado, M. (2008). "Los confines de la impostura. Reflexiones sobre el trabajo etnográfico entre minorías religiosas". Revista de Dialectología y Tradiciones Populares, LXIII-1: 147-172.

Carreras, J. J. \& Forcadell, C. (2003). Usos públicos de la historia. Madrid: Marcial Pons.

Charriez Cordero, M. (2012). "Historias de vida: Una metodología de investigación cualitativa". Revista Griot, 5-1: 50-67.

Del Río Sánchez, A. \& Valcuende del Río, J.M. (2008(. "Historias de vida y microbiografías. Una aproximación metodológica”. En Acosta Bono, G. - Del Río Sánchez, A. - Valcuende 
del Río, J. M. (Coords). 2008. La recuperación de la memoria histórica. Una perspectiva transversal desde las ciencias sociales. Sevilla: Fundación Pública Andaluza Centro de Estudios Andaluces.

Dewalt, K.M. \& Dewalt, B.R. (2002). Participant Observation: A Guide for Fieldworkers. New York: Altamira Press.

Díaz Sánchez, M., (2016). "La emigración como estrategia de escape a la represión social, política y económica de la dictadura franquista. El caso de los murcianos en Barcelona (19391950)". Dictatorships and Democracies. Journal of History and Culture. 4: 9-40.

Domanska, E. (2006). "The return to things". Archaeologia Polona, vol. 44: 171-185.

Douglas, L. (2014). "Mass graves gone missing: Producing knowledge in a world of absence". Culture \& History Digital Journal, 3-2.

Dumont, G. (2012). "Multiplicidades móviles, dibujo de una pluralidad situacional”. Encrucijadas. Revista crítica de ciencias sociales, 4: 66-80.

Etxeberría, F. (2004). "Panorama organizativo sobre antropología y patología forense en España. Algunas propuestas para el estudio de fosas con restos humanos de la Guerra Civil española de 1936. 183-219. En Silva, e. - Esteban, A. - Castañas, J. - Salvador: (eds.). La memoria de los Olvidados. Valladolid: Ámbito Ediciones.

Falzón, M. A. (2009). "Multi-sited Ethnography: Theory, Praxis and Locality in Contemporary Research". En Falzón, M. A. (ed.). Multi-Sited Ethnography Theory, Praxis and Locality in Contemporary Research. Farnham: Ashgate.

Fernández de Mata, I. (2006). “The 'Logics’ of Violence and Franco’s Mass Graves. An Ethnohistorical Approach". International Journal of the Humanities 2:3: 2527-2535.

Fernández de Mata, I. (2016). Lloros vueltos puños. El conflicto de los 'desaparecidos' y vencidos de la Guerra Civil española. Granada: Comares.

Ferrándiz, F. (2011). Etnografías contemporáneas. Anclajes, métodos y claves para el futuro. Barcelona: Anthropos.

Goldhagen, D. J. (1997). Los alemanes corrientes y el Holocausto. Madrid: Taurus.

Hammersley, M. \& Atkinson: (1994). Etnografía. Métodos de investigación. Barcelona: Paidós.

Hudson, J. M. \& Bruckman, A. S. (2004). "The Bystander Effect: A Lens for Understanding Patterns of Participation". Journal of the Learning Sciences, 13:2: 165-195.

Juliá, S. (Coord.). (1999). Víctimas de la guerra civil. Madrid: Temas de Hoy.

López García, J. - Ferrándiz, F. (Coords.) (2010). Fontanosas, 1941-2006. Memoria de carne y hueso. Ciudad Real: Imprenta provincial.

Marcus, G. \& Fischer, M. M. J. (2000). La antropología como crítica cultural. Un momento experimental en las ciencias humanas. Buenos Aires: Amorrortu.

Marcus, G. (2001). "Etnografía en/del sistema mundo: el surgimiento de la etnografía multilocal". Alteridades 11(22): 111-127.

Montero Gutiérrez, J. (2010): "Exhumando el legado material de la represión franquista. De la percepción social a la encrucijada jurídica y patrimonial”. En J. Almansa Sánchez (ed). Recorriendo la Memoria/Touring Memory. BAR IS, 2168. Oxford: Archaeopress.

Montero Gutiérrez, J. (2011): "La Arqueología al servicio de la gestión de la Memoria Histórica: la exhumación de fosas comunes de la Guerra Civil española en la provincia de Burgos". Actas de las II Jornadas de Jóvenes en Investigación Arqueológica (Madrid, 2009). Zaragoza: Libros Pórtico.

Narotzky, S. (2002). "Reivindicación de la ambivalencia teórica: la reciprocidad como concepto clave". ÉNDOXA: Series Filosóficas, 15: 15-29.

Parker Pearson, M.G. (1999). The Archaeology of Death and Burial. Stroud UK: Sutton Publishing Ltd.

Peiró Martín, I. (2004). "La era de la memoria: reflexiones sobre la historia, la opinión pública y los historiadores". Memoria y Civilización, 7, 243-294. Aquí 248 y 249.

Pujadas, J. J. (1992). El método biográfico. El uso de las historias de vida en ciencias sociales. Madrid: CIS.

Pujadas, J. J. (2000). "El método biográfico y los géneros de la memoria”. Revista de Antropología Social, 9: 127-158. Aquí, pág. 139.

Renshaw, L. (2010). “The scientific and affective identification of Republican civilian victims from 
the Spanish Civil War". Journal of Material Culture, 15(4): 449-463.

Renshaw, L. (2011). Exhuming Loss: Memory, Materiality and Mass Graves of the Spanish Civil War. Walnut Creek: Left Coast Press.

Renshaw, L. (2013). "The dead and their public. Memory campaigns, issue networks and the role of the archaeologist in the excavation of mass graves". Archaeological Dialogues, 20(1): $35-47$.

Ruiz Olabuénaga, J. I. (2003). Metodología de la investigación cualitativa. Bilbao: Universidad de Deusto.

Velasco, H. y Díaz de Rada, A. (1999). La lógica de la investigación etnográfica. Madrid: Trotta.

Varisco, D. (2002). "September 11: Participant Webervation of the "War on Terrorism"”. American Anthropologist, 104: 934-938. 